\title{
LA EPISTEMOLOGÍA CONSTRUCTIVISTA Y LA DIDÁCTICA DE LAS CIENCIAS: ¿COINCIDENCIA O COMPLEMENTARIEDAD?
}

\author{
MORENO ARMELLA, LUIS E. ${ }^{1}$ y WALDEGG, GUILLERMINA ${ }^{2}$ \\ ${ }^{1}$ Departamento de Matemática Educativa, Cinvestav. IPN. \\ ${ }^{2}$ Sección de Metodología y Teoría de la Ciencia, Cinvestav. IPN.
}

\begin{abstract}
SUMMARY
To a large extent, criticisms to constructivists epistemologies, in particular to Piaget's theory, have come from fields like psychology and didactics, not from epistemology itself. Our goal is to show that the power of piagetian theory comes from its epistemological contents. One must not look for this power in education for its own sake. This does not mean that epistemology and educational theories are unrelated but that each one has to define its domain of applicability.
\end{abstract}

\section{INTRODUCCIÓN}

Las investigaciones en didáctica que indagan sobre los procesos de enseñanza y aprendizaje de las ciencias han alcanzado en las últimas décadas niveles de consolidación considerables, lo que hace necesario caracterizar los marcos conceptuales que determinan nuestras explicaciones acerca de los fenómenos vinculados a la educación científica.

Los métodos de enseñanza, el diseño de estructuras curriculares, los textos y materiales didácticos y la práctica dentro del aula han estado -porque no puede ser de otra manera- inspirados en las concepciones científicas de los educadores. Si consideramos el dominio del paradigma empirista de la ciencia en buena parte de nuestro siglo, no es extraño ver que las ciencias hayan sido tratadas en la escuela como un cuerpo inalterable de conocimientos preexistentes. Bajo este paradigma epistemológico, el papel del profesor y de quienes producen los planes de estudio, los textos y los materiales didácticos ha consistido en diseñar estrategias curriculares y didácticas, que faciliten a los estudiantes la asimilación del conocimiento transmitido. La concepción que subyace a esta actividad supone que existe una relación mecánica entre transmisión y asimilación.

Durante muchos años hemos aceptado una concepción educativa que no distingue entre entrenamiento y enseñanza. Hemos supuesto que el conocimiento es un bien que debe ser entregado al estudiante por medio de una práctica didáctica preestablecida, para ello se han sobreestimado actividades como la memorización, la repetición y la realización de tareas rutinarias. Sin embargo, ahora nos damos cuenta que resolver problemas en el sentido amplio, como lo establecen la mayoría de los propósitos explícitos de la educación científica en todos los países, exige del estudiante una comprensión que va más allá de este primer nivel. Para lograrlo, sabemos que el estudiante debe llevar a cabo otras actividades, distintas y más complejas, que incluyen no sólo una reflexión sobre sus operaciones, sino una reflexión sobre su reflexión. La forma de comprensión que resulta de esta actividad metacognitiva, sabemos 
-lo dicen los estudios realizados a este respecto- que no puede ser transmitida, en el sentido tradicional, al estudiante. Es algo que él tiene que construir con sus propios medios y que el profesor debe reconocer y propiciar.

La concepción mecanicista, que supone que al generarse un proceso de emisión de información por parte del profesor se activa automáticamente un proceso de asimilación de dicha información por parte del estudiante, tiene una vieja historia. Que las cosas no son así es algo que se puede constatar mediante la presencia, en el campo de conocimientos del estudiante -a la hora del examen, por ejemplo-, de elementos que no estaban presentes en el discurso de enseñanza del profesor.

¿Cuáles son las alternativas a este estado de cosas? Responder a este interrogante es uno de los propósitos principales de los estudios sobre la enseñanza de las ciencias. Uno de los puntos de partida de estas indagaciones está en las ciencias mismas. Empero, el conocimiento científico, si bien es necesario, no es suficiente para la caracterización de una disciplina cuyo objeto de estudio es la enseñanza y el aprendizaje de la ciencia y no la ciencia misma. Una condición indispensable para tal caracterización, la constituye la interacción continua con el sistema educativo y con los actores -alumnos y maestros- del proceso.

\section{LA ALTERNATIYA CONSTRUCTIVISTA EN EPISTEMOLOGIA}

Hemos insistido anteriormente en que las concepciones sobre la ciencia que tiene el educador modelan y modulan sus prácticas pedagógicas. Estas concepciones son, con frecuencia, implícitas y, por tanto, caen fuera de la esfera de los esfuerzos conscientes del profesor por identificar las posibles causas de los fracasos de sus alumnos. De ahí que resulte importante la toma de conciencia, por parte del educador, de sus convicciones sobre la naturaleza del conocimiento científico, sobre cómo éste se genera, sobre las relaciones entre el conocimiento y la realidad y entre las distintas manifestaciones del saber científico, de modo que el educador pueda emplear, de manera explícita, estas ideas en el diseño de su acción pedagógica.

La epistemología, en su versión contemporánea, se propone el estudio de la naturaleza del conocimiento científico y de las circunstancias de su producción. Ya desde los tiempos de la antigüedad clásica griega era dominante el pensamiento epistemológico realista que concibe el conocimiento como una copia de la realidad: el conocimiento se considera el reflejo -como la imagen en un espejo- de ese mundo externo que existe con independencia del observador. El enfoque tradicional de la enseñanza tiene raíces profundas en esta epistemología realista (Moreno-Waldegg, 1992) que se complementa armónicamente con el paradigma empirista; bajo este punto de vista, la actividad del sujeto que trata de conocer (el sujeto cognoscente) queda subordinada al objeto de su conocimiento y su actividad -primordial- mente perceptual- y sólo puede producir un conocimiento que es reflejo fiel de una realidad externa estructurada.

Si bien esta concepción realista-empirista del conocimiento resulta ser una especie de «respuesta espontánea» del hombre común ante las preguntas sobre la naturaleza del conocimiento, no ha estado, desde sus primeras manifestaciones en la Grecia antigua, libre de cuestionamientos. En el siglo $\mathrm{V}$ aC, los escépticos hicieron evidente la imposibilidad lógica de establecer la «verdad» de un conocimiento, ya que la necesaria comparación de ese conocimiento con la parte de la realidad que supuestamente representa implica un nuevo acto de conocimiento, que tendría también que ponerse a prueba para demostrar su verdad. Ésta sólo es la primera de una larga cadena de objeciones a la que se tuvieron que enfrentar quienes defendían el realismo y el empirismo epistemológico.

Reaccionando al punto de vista realista-empirista, Kant (1724-1804) postula en su Crítica de la razón pura que, cuando el sujeto entra en contacto con su objeto de conocimiento, recibe impresiones sensibles que somete a un proceso organizador, mediante estructuras cognitivas innatas. Lo que resulta es el conocimiento. Así como el líquido adopta la forma del recipiente que lo contiene, así también las impresiones sensoriales adoptan las formas que les son impuestas por las estructuras cognitivas que las procesan; el resultado de este procesamiento es el conocimiento. De esta manera, Kant nos advierte sobre las condiciones de posibilidad del conocimiento objetivo: para alcanzarlo se requiere de ciertas formas innatas de sensibilidad, éstas son el espacio, el tiempo, la causalidad, la permanencia del objeto. En otros términos, aunque la realidad existe con independencia del sujeto, el conocimiento que éste puede tener de aquélla está mediado por la capacidad cognoscitiva intrínseca del sujeto.

Hay dos consecuencias fundamentales del enfoque kantiano: la primera es que el conocimiento deja de ser concebido como representación de la realidad externa y, en su lugar, es visto como resultado inseparable de las experiencias del sujeto y de su actividad cognoscitiva; en la segunda, el sujeto deja de ser cognitivamente pasivo frente al objeto de su conocimiento. El sujeto da estructura a sus experiencias. Esto ya lo habían adelantado las corrientes racionalistas, pero a costa de llegar al extremo de poner todo el peso de la construcción del conocimiento en el sujeto cognoscente, marginando el objeto. La posición kantiana inauguró un nuevo modo de conceptualizar la actividad cognoscitiva. Sobre ella trabajaría Piaget dos siglos más tarde.

Para Piaget, el sujeto se acerca al objeto de conocimiento dotado de ciertas estructuras cognitivas previamente construidas (no innatas), mediante las cuales asimila el objeto de conocimiento. Esta asimilación activa una transformación (acomodación) de su aparato cognitivo, de modo que, en el siguiente acercamiento, su «lectura» del objeto será otra, pues, como resultado de la primera, las estructuras cognitivas del sujeto se han modificado. 
Con ello se establece una diferencia central con la posición de Kant: las estructuras cognitivas piagetianas son estructuras que se generan y evolucionan en el tiempo.

Las estructuras cognitivas del sujeto se van transformando. Con el paso del tiempo, el sujeto se va encontrando en posesión de un aparato cognitivo cada vez más adaptado a su entorno. Por ejemplo, la lógica de un niño pequeño es cualitativamente distinta a la lógica de un adulto; como consecuencia, la imagen del mundo del niño es distinta de la imagen del adulto; sin embargo, en ninguno de los dos casos, la imagen del mundo es una copia de una realidad que «esté allí», estructurada, lista para ser asimilada. La dimensión constructivista de la epistemología piagetiana se refiere a que el sujeto va construyendo sus sucesivas versiones del mundo al mismo tiempo que construye sus propias estructuras cognitivas. Su conocimiento no es copia de una realidad externa a él, sino resultado de la estructuración de sus propias experiencias.

Una idea primordial que subyace a la obra de Piaget es la de evolución. A ella corresponde un punto de vista filosófico y científico que consiste en fijar nuestra atención en la naturaleza dinámica y cambiante de las cosas y estudiar, entonces, sus transformaciones a lo largo del tiempo. En esencia, este punto de vista, dominante ya a fines del siglo pasado, fue una consecuencia duradera de la obra de Darwin.

\section{UNA EPISTEMOLOGÍA EXPERIMENTAL}

Piaget quiso que la epistemología estuviese dotada de mecanismos de control sobre sus afirmaciones. La historia de la ciencia (concebida como laboratorio epistemológico) y la psicología le darían los elementos para diseñar el dominio experimental de su versión de esta disciplina.

El objetivo de la epistemología genética es la explicación del conocimiento científico; su base experimental, la constituye la historia de la ciencia y ciertos experimentos psicológicos, que quedan enmarcados en la llamada psicología genética, desarrollada para tales fines por Piaget y su escuela ginebrina. Describiremos ahora las relaciones entre las tesis epistemológicas centrales de la teoría piagetiana y los «experimentos psicológicos» diseñados como parte del dominio empírico correspondiente, al tiempo que haremos algunas anotaciones desde la perspectiva educativa.

Piaget siempre estuvo bajo la fuerte influencia de la ciencia de su tiempo (esto ya es evidente en su artículo Las dos direcciones del pensamiento científico, de 1929). Su epistemología está pensada alrededor de las categorías básicas de la ciencia: el espacio, el tiempo, la causalidad, el principio de conservación de la materia, el número, etc. Piaget realizó investigaciones decisivas sobre estas categorías, desde la perspectiva de la historia de las ideas, que lo llevaron a una explicación de la razón profunda de la existencia de un pensamiento racional. Pero consideró necesario dar una mayor sustentación empírica a sus aseveraciones de orden epistemológico. Entonces, su «laboratorio epistemológico», constituido por la historia de la ciencia, se vio ampliado con sus investigaciones psicogenéticas ${ }^{1}$. De ahí extrae una información fundamental: existe una lógica del niño, cualitativamente distinta a la lógica del adulto. Este resultado está en el corazón de su teoría, pues le permitió explicar el origen operatorio de las estructuras lógicas (punto débil del empirismo) además de verificar una vieja hipótesis sobre la existencia de una «lógica de la acción» (la del niño pequeño) que sirve como punto de partida para la construcción de la lógica del pensamiento adulto. Para Piaget, el pensamiento es una acción que se lleva a cabo internamente; para su descripción requiere de un análogo interiorizado del movimiento y de la percepción. La función simbólica hace posible esta nueva forma de acción: se comienza con las representaciones simples del mundo sensoriomotor y de allí se llega a las operaciones concretas que se apoyan sobre aquellas primeras representaciones. El periodo de las operaciones concretas tiene como núcleo la posibilidad de aplicar, por parte del sujeto, algún principio de conservación. Debe entenderse que esto ocurre siempre dentro de un contexto y que el éxito en la aplicación de un principio de conservación en dicho contexto no significa que el sujeto ya pueda aplicar tal principio en cualquier otra situación. Lo que le interesa a la epistemología genética, como tal, es que la posibilidad de aplicar un principio de conservación revela un cambio cualitativo ${ }^{2}$. En la etapa final del proceso (que es muy largo, complejo y altamente nohomogéneo) aparecen las formas complejas de organización del pensamiento científico. El núcleo de la etapa de las operaciones formales lo constituye la posibilidad del pensamiento hipotético-deductivo, es decir, la posibilidad de razonar a partir de hipótesis. Volvamos a insistir, la posibilidad significa que, en una situación determinada, el sujeto es capaz de esta forma compleja de razonamiento. Es allí, en esa posibilidad, donde se encuentra el valor epistemológico que interesa a la epistemología genética ${ }^{3}$.

El análisis de la génesis histórica de las categorías básicas del pensamiento científico permitió a Piaget la tematización (es decir, el estudio sistematizado) de la objetivación y del aumento de claridad conceptual (que podemos asociar a un aumento de rigor) en el desarrollo de las ciencias. La actividad de la comunidad científica va llevando al conocimiento, en una época determinada, a un mayor nivel de objetividad. La objetividad no es, pues, una característica del conocimiento que cae ya preformado ante los ojos de la comunidad.

Pero hablar de la actividad de los científicos es hablar de un nivel de desarrollo avanzado. Si de lo que se trata es de investigar el proceso de construcción del conocimiento científico, la perspectiva evolutiva indica que hay que ir hacia atrás, hacia las etapas anteriores, ya que la realidad de un proceso evolutivo no la descubre ninguna de sus etapas en particular, sino el proceso en su totalidad. Al hacerse difuso el seguimiento de las ideas en las épocas más tempranas, se desemboca en la psico- 
génesis como parte de una estrategia que permite la construcción de un modelo del sistema cognitivo. Se trata entonces de indagar los orígenes del funcionamiento cognitivo de un sujeto frente a problemas diseñados a partir de las categorías como espacio, tiempo, conservación de la materia, conservación de la cantidad, etc.

Sin embargo, el largo y arduo trabajo requerido por la investigación psicogenética absorbió casi la mayoría del tiempo y las energías de Piaget (durante más de 50 años); no fue fácil descubrir, por ejemplo, las raíces de la lógica allí en el dominio de la inteligencia sensoriomotriz. Las consecuencias no se hicieron esperar: dio la impresión de que su trabajo estaba orientado completamente a la psicología, lo que fue en perjuicio de las interpretaciones epistemológicas de su trabajo experimental.

Es pertinente señalar en este momento que la epistemología genética no es una teoría inductiva, extraída de las «evidencias» suministradas por las indagaciones psicogenéticas ni por las histórico-críticas. Más bien, de lo que se trataba era de explorar la posibilidad de comprobación, desde estas dos dimensiones experimentales, de los hechos determinados por la teoría. Este punto de vista tiene una importancia particular con respecto a la teoría psicogenética que ha sido el blanco preferido de quienes confunden esta teoría con una psicología del aprendizaje, en el sentido más tradicional del término. Así pues, lo que Piaget observa, cuando observa el funcionamiento cognitivo de los niños, son hechos determinados por su teoría.

Un ejemplo importante del uso epistemológico que Piaget da a sus investigaciones psicogenéticas, lo constituye la forma en que refuta el empirismo. Sus investigaciones muestran que la conservación del número de elementos de una colección de cuentas, por ejemplo, no es extraído directamente de las agrupaciones de cuentas, no depende de la disposición espacial de los elementos de dicha colección. Es resultado de una construcción que el sujeto hace a partir de una reflexión sobre sus propias acciones.

Aunque la teoría piagetiana señala que los avances cognitivos del individuo suponen adaptaciones a su entorno, físico y social, sus esfuerzos van encaminados básicamente a explicar cómo el sujeto puede dar sentido a un mundo genérico que se describe desde las categorías básicas del pensamiento científico. El sujeto, que explora en ese mundo armado fundamentalmente con su lógica de la acción, nos ofrece la oportunidad de observar cómo se articulan los conceptos a lo largo del desarrollo de su visión del mundo.

Piaget postula que este tipo de observación psicogenética permite construir un modelo de desarrollo del desarrollo científico (el modelo «original» no lo podemos reconstruir dado lo fragmentario de la información histórica o prehistórica que tenemos a nuestra disposición).

Hay dos observaciones que deben reiterarse: la primera, que el sujeto psicológico de Piaget no es el sujeto de las teorías psicológicas tradicionales. Es un sujeto que responde a preguntas sobre las categorías de la ciencia: espacio, tiempo, conservación de la materia, etc. Un sustrato cognitivo común, un modelo genérico de desarrollo, por lo menos dentro de las culturas occidentales (Bringuier, 1985, p. 100).

La segunda observación viene en forma de pregunta: ¿Cómo evaluar esta hipótesis? Piaget espera que esto se haga de igual forma a como se hace en las ciencias naturales: estimando la capacidad explicativa de su teoría. Esto es indispensable, reiteramos, porque su teoría no tiene pretensiones inductivistas ${ }^{4}$.

El sujeto que interesa a Piaget es aquél que construye la conservación del objeto, que descubre a partir de una reflexión sobre sus acciones que la cantidad de arcilla no cambia aunque cambiemos la forma del material, mostrando con ello que ha accedido a una forma de pensar nueva: antes no podía resolver el problema que se le planteaba con la arcilla; ahora ya puede. Después, mostrará que accede a otra forma de razonamiento, cuando sea capaz de prescindir de cualquier sustrato material como sustento de su pensamiento (no se discute en ese momento cómo llega a ser capaz, sólo que llega a serlo). Ese sujeto (el sujeto epistémico) es una abstracción, como lo es el principio de inercia de la dinámica de Galileo y la ley de gravitación universal de Newton; su existencia supone condiciones ideales y, por tanto, imposibles de encontrar en las situaciones de la vida cotidiana.

\section{LÓGICA Y CULTURA}

La teoría del sujeto escolar no puede extraerse mecánicamente, por todo lo que esto implica (o deja de implicar) de la teoría psicogenética de Piaget. El que muchos de sus seguidores lo hayan intentado ha costado a Piaget un número considerable de críticas desde la psicología. Piaget, como Newton antes que él, hace hipótesis, pero estas hipótesis son orientadas a su teoría epistemológica.

Para Piaget, el conocimiento es un fenómeno social que sufre procesos de cambio tanto desde el punto de vista individual como de la historia de la ciencia. Hay que comprender primero cómo se dan esos procesos de cambio, para después poder identificar cuáles son los mecanismos que los conducen.

Para apreciar la dimensión social del conocimiento, comencemos con un ejemplo: el número. Tenemos hoy a nuestra disposición una obra monumental que documenta con precisión y profundidad conceptual la historia de este concepto. Nos referimos a la Histoire universelle des chiffres (Ifrah, 1994). ¿Qué podemos aprender de esta historia? Que ya desde épocas muy tempranas el ser humano ha construido diversas representaciones para enfrentarse al problema de la numerosidad, y que todas ellas lo han conducido a la noción de número:

«Hubo un tiempo, cuando los hombres no sabían contar [...] el concepto de número debía estar revestido, en sus espíritus, del aspecto de una realidad concreta, indiso- 
ciable de la naturaleza de los objetos, que se reducía a una suerte de percepción directa de la pluralidad material [...] Nuestros lejanos ancestros, muy probablemente, estaban incapacitados mentalmente para concebir los números [en abstracto], probablemente no tenían conciencia de que el día y la noche, las alas de un pájaro, los ojos, las orejas, los brazos, las piernas de un humano, presentan una característica común, que es precisamente aquella de «ser dos» [...] Los estudios realizados con niños pequeños así como los análisis etnográficos llevados a cabo con pueblos «primitivos» contemporáneos dan mayor fuerza a estas [interpretaciones]...» (Ifrah, op. cit., p. 21).

Los seres humanos poseen pocos instintos. El proceso evolutivo casi «destruyó» la estructura instintiva humana. Pero, a cambio de eso, tenemos la capacidad potencial de asimilar, de reconstruir los logros intelectuales que nos han precedido. Desde luego, para eso contamos con el medio social al que pertenecemos. Contamos con la educación.

La infancia corresponde a una etapa en la que se realiza un inmenso trabajo de elaboración y recreación. Es necesaria la infancia para la realización de una lógica de la acción, que se usa para actuar sobre los dominios inmediatos de nuestra percepción. La lógica corresponde a la coordinación general de las acciones. Más adelante, con el progreso evidente de la capacidad semiótica, se crean las posibilidades para la internalización plena de esta lógica, transformándose entonces en una lógica que actúa sobre formas simbólicas. Es la lógica formal del adulto, cualitativamente distinta a la lógica del niño, como ha sido demostrado fehacientemente (Piaget, 1987).

La posibilidad de la traducción del cero «de antes» (el cero maya, por ejemplo) al cero «de ahora», como cualquier problema de traducción y comunicación, es posible debido a que las diversas culturas comparten estructuras lógicas de base. Ésta es una respuesta piagetiana que se ha abierto espacio debido a su fuerza explicativa y también a la tarea, estrechamente vinculada, de comprobación experimental en diversas culturas ${ }^{5}$.

Durante años se han realizado, en diversas partes del mundo, un número considerable de investigaciones cuyo propósito ha sido «verificar» o «refutar» la teoría de Piaget sobre el desarrollo cognitivo en los más diversos entornos culturales. La secuencia del desarrollo cognitivo (Moreno 1996, para una descripción sucinta) se encuentra en todas partes. Cambia, eso sí, el ritmo de desarrollo de las diferentes nociones (aquéllas que interesan a la epistemología) de acuerdo con el entorno cultural. Así, por ejemplo, en sociedades nómadas que viven de la caza, hay un mayor desarrollo de las habilidades espaciales, que claramente son más valoradas en estas organizaciones sociales. Allí mismo, se valoran menos las nociones relativas a la cantidad, aunque no están ausentes. En las sociedades sedentarias ocurre justamente lo opuesto. Los factores ecoculturales no afectan, empero, el orden de aparición de las etapas. Afectan, eso sí, los niveles de competencia y desempeño (Dasen, 1988, p. 266). El trabajo de campo ha permitido mostrar que hay una serie de procesos cognitivos de base que sufren afectaciones culturales. El desarrollo cognitivo, de acuerdo con los datos que ha arrojado hasta ahora la investigación en diferentes culturas, no es ni totalmente universal ni totalmente cultural. Para la educación hay aquí una sugerencia poderosa: hay que tener en cuenta ambas dimensiones de la cognición: lo universal y lo cultural.

El conocimiento cotidiano (Dasen, 1988, p. 267) está vinculado a los contextos particulares y presenta características más orientadas a la eficiencia de las tareas que a la conceptualización. En este dominio, en lugar de hablar de conocimiento universal o específico, se habla de conocimiento generalizable o particular. Recordemos que el contexto, en el que está enraizada toda actividad humana, no es tanto una serie de estímulos que afectan a las personas sino, más bien, una red de relaciones que dan significado a la acción.

Bishop (1988, p. 60) da cuenta de un interesante trabajo que Lancy realizó en Nueva Guinea y que lo llevó a desarrollar una teoría del desarrollo cognitivo para explicar sus resultados. Lancy llegó a la conclusión de que el desarrollo cognitivo de las sociedades pasa esencialmente por tres etapas.

La primera corresponde a las etapas sensoriomotora y preoperacional de Piaget, con algunas características de la etapa de las operaciones concretas. Lancy argumenta que «los logros de esta etapa son compartidos por todos los seres humanos» (ídem). Llega incluso a sugerir que es la etapa en donde la programación genética surte los mayores efectos.

De la segunda etapa, nos dice Lancy: «Lo que ocurre con la cognición tiene mucho más que ver con la cultura y menos con la genética» (ídem). Es la etapa en la cual, las distintas culturas se interesan en distintos tipos de fenómenos.

Aunque diferentes entornos culturales impulsen el desarrollo cognitivo en diferentes direcciones, no ocurre que se produzcan modos de pensar totalmente divergentes. En las diferentes culturas se cuenta, se mide, se desarrollan conceptos geométricos, se juega (de acuerdo con reglas) y se desarrollan formas de explicar.

Lancy sostiene que es necesaria la consideración de una tercera etapa, donde se considera el nivel metacognitivo. Dice lo siguiente:

«En esta etapa, además de desarrollar estrategias lingüísticas y cognitivas, los individuos adquieren teorías sobre el lenguaje y la cognición. Aprenden a distinguir sus conocimientos de acuerdo a sus propósitos [...]» (Bishop, op. cit., p. 61).

Según este enfoque, la etapa de las «operaciones formales» de Piaget representa la teoría particular del conocimiento que es enfatizada por la cultura occidental y que alcanza su manifestación más acabada en la ciencia actual. Esto explicaría, al menos parcialmente, las difi- 
cultades que tienen las culturas y tradiciones no occidentales por desarrollar autónomamente una ciencia que encarna los valores occidentales. Este desarrollo requiere de un trabajo previo de «traducción» que, aunque posibilitado por la existencia de una lógica subyacente común, requiere de esfuerzos considerables por parte de las culturas no occidentales. Éste es un foco de alerta para los sistemas educativos de los llamados países del Tercer Mundo.

Resultados análogos arroja el trabajo de Saxe (Hameline-Voneche [eds.], 1996) sobre el número y la medición. Aquí también las características esenciales de la teoría piagetiana se revelan como universales. Podría decirse que el mensaje que se desprende de estas experiencias (las citadas aquí y muchas otras análogas) es que más allá de la diversidad cultural está la unidad de la especie humana. Somos los mismos y somos diferentes. Las diferencias culturales quedan registradas al nivel de las diversas formas de representación (Bourges, 1998). Los valores culturales encuentran su camino a través de tales representaciones.

En su libro Psicogénesis e historia de la ciencia, Piaget y García expresan su punto de vista con relación al papel que desempeña el entorno social:

«[...] la acción, excepto al comienzo mismo del período sensoriomotriz, no tiene lugar solamente en función de impulsos internos [...] bien pronto en la experiencia del niño, las situaciones con las cuales se enfrenta son generadas por su entorno social [...] no se asimilan objetos "puros". Se asimilan situaciones en las cuales los objetos desempeñan ciertos papeles y no otros. Cuando el sistema de comunicación del niño con su entorno social se hace más complejo y más rico, y particularmente cuando el lenguaje se convierte en el medio dominante, lo que podríamos llamar la experiencia directa de los objetos comienza a quedar subordinada al sistema de significaciones que le otorga el medio social. El problema que aquí surge para la epistemología genética es explicar cómo queda la asimilación, en dichos casos, condicionada por el sistema social de significaciones, y en qué medida la interpretación de cada experiencia depende de ellas» (p. 228, subrayado nuestro).

A partir de la revisión de la obra piagetiana, se ve la necesidad de establecer una clara distinción entre los problemas de orden epistemológico y de aquéllos que corresponden al dominio de la educación. Es importante que podamos dilucidar cómo aquello que aparece como un proceso privado de construcción epistémica es, en realidad, permeado por la cultura. En los ámbitos educativos, esto implica el estudio de la interacción entre estudiantes, profesor, textos y demás elementos portadores de información que puedan ser empleados en el proceso. Lo que podemos llamar «conocimiento objetivo» es consecuencia de dos actividades centrales: la cooperación y la coordinación de los distintos puntos de vista. Ya en 1925, Piaget (p. 46) afirmaba que, con la necesidad de comunicar y discutir, aparece la necesidad de demostrar y verificar; con la discusión aparece la capacidad de deducir y de razonar verbalmente. En suma, la socialización del pensamiento entraña un progreso lógico innegable. Pero es importante añadir de inmediato que este efecto de socialización no es mecánico ni automático: el niño se socializa en la medida en que «co-ordina» sus puntos de vista (lo que lo conduce al descentramiento cognitivo) y alcanza un nivel de cooperación con los demás. Piaget nos dice:

«Todo pensamiento lógico está socializado, porque implica la posibilidad de comunicación entre individuos. Este intercambio interpersonal se realiza mediante correspondencias, fusiones, intersecciones y reciprocidades, es decir, mediante operaciones. Así pues, las operaciones realizadas dentro de los individuos se identifican con las operaciones realizadas entre los individuos que constituyen [así] la "co-operación" en el sentido propio y etimológico de la palabra» (Respuesta a Vygotski,1995, p. 194).

La idea de que el sujeto nace con su «banco cognitivo» completo no goza hoy día de mucho apoyo. Tampoco es muy acogida la idea contraria, es decir, que todo el conocimiento del sujeto es adquirido. Un término medio se impone: el sujeto nace con la potencialidad de interactuar con su entorno, de ser sensible a él de diversas formas y, a partir de allí, desarrollar sus estructuras cognitivas a través de la interacción con el medio físico $\mathrm{y}$, sobretodo, con el medio social. Hay dos elementos en esta expresión que son claves: el sujeto construye su conocimiento y desarrolla los instrumentos cognitivos necesarios, para ello el sujeto necesita la interacción. El tipo de interacción que necesita va cambiando en una relación dialéctica con su propio desarrollo. Estos dos términos son necesarios para el diseño de una perspectiva cognoscitiva de la educación. Tenerlos presentes favorece una toma de conciencia sobre el papel activo del sujeto en la elaboración del conocimiento y la necesidad de considerar, en dicho diseño, la dimensión social del aprendizaje.

Los procesos de enseñanza y de aprendizaje de las ciencias no pueden evitar las consideraciones, a fondo, sobre la naturaleza del conocimiento que se quiere enseñar. Esto implica estudios complementarios de naturaleza sociogenética y psicogenética que nos permitan apreciar las componentes individual y cultural del desarrollo del conocimiento. Podríamos citar, a modo de ejemplo, el desarrollo de las geometrías noeuclidianas. Durante muchos siglos, los matemáticos estuvieron convencidos de que, con un cierta dosis de ingenio, sería posible demostrar la veracidad del postulado de las paralelas. Esa creencia no formaba parte de la geometría como tal; estaba enraizada en una concepción del mundo que aquellos matemáticos compartían a pesar de provenir de culturas muy distintas. De allí podemos concluir que las relaciones entre los procesos cognitivos como tal y las culturas en que toman cuerpo son complejas; la historia de la ciencia está llena de ejemplos que ilustran la complejidad de estas relaciones.

Otro ejemplo: la concepción geocéntrica del sistema solar. Distintas culturas han compartido una visión del mundo que pone la Tierra en el centro del Universo. 
La han usado para predecir eclipses. Aunque la interpretación del eclipse en sí mismo haya variado, la lógica subyacente a los cálculos ha sido la misma. En otros casos, consideraciones culturales o ideológicas han determinado el campo de interés de los científicos. Pero los métodos, aunque distintos, están construidos con una lógica que hace posible comprenderlos, aun desde otras concepciones del mundo que pudieran ser divergentes ${ }^{6}$.

\section{EL CONOCIMIENTO ESTRATIFICADO}

La tesis epistemológica piagetiana que afirma que el conocimiento es una construcción sucesiva, individual y social, de la realidad experiencial de los sujetos tiene una consecuencia decisiva para la enseñanza de las ciencias: los niños y jóvenes inician su formación científica escolar con un acervo propio de explicaciones de los fenómenos naturales, elaborado sobre la base de las experiencias con su mundo físico, social y cultural. Estas explicaciones son, a menudo, incompatibles con las explicaciones de la ciencia establecida y constituyen el factor aislado más importante que dificulta la enseñanza y el aprendizaje de los conceptos científicos.

Si adoptásemos una perspectiva lineal de la apropiación de una ciencia, en la cual el conocimiento «viejo»va siendo sustituido por un conocimiento «nuevo», podríamos perder de vista que el sujeto puede tener (y de hecho los tiene) diversos enfoques conceptuales sobre un mismo tema. Esos enfoques dependen de las circunstancias en las cuales se manifiesta el conocimiento, es decir, de su contexto. Por ejemplo, un físico profesional puede pedir a una persona, en un contexto coloquial, que cierre la ventana de una habitación «para que no se meta el frío», aunque sepa, en estricto sentido, que está invirtiendo el proceso físico al emplear esa estructura lingüística. El aprendizaje ocurre mediante construcciones paralelas, relativas a contextos específicos (Driver et al., 1994). Los individuos no piensan de una única manera sobre un tema: van adoptando perfiles conceptuales de acuerdo con los dominios específicos que son objeto de sus indagaciones.

Durante el aprendizaje de una ciencia, los estudiantes son introducidos en un mundo conceptual y simbólico. Este mundo no lo construye el estudiante solo: necesita la interacción con los compañeros y maestros. Entonces, al poner en juego sus concepciones previas y las que se va construyendo, alcanza a vislumbrar las limitaciones de sus propias ideas. El proceso de asimilación y acomodación de las distintas estructuras conceptuales de la ciencia incluye los procesos dialógicos. Esto es fundamental tanto desde el punto de vista cognitivo como desde el punto de vista escolar.

Las investigaciones sobre la estratificación del conocimiento y la contextualización han dado lugar a una literatura considerable sobre el tema. Los estudios interculturales sobre concepciones alternativas, preconcepciones y conceptos científicos se han incrementado du- rante la última década (Thijs y Van Der Berg, 1995, y las referencias citadas en este artículo central).

El aprendizaje de una ciencia implica un proceso de iniciación a las ideas y prácticas de una comunidad científica. El aprendizaje científico se puede ver como la iniciación a una nueva cultura o como el proceso de aprendizaje de una segunda lengua. En los estudios interculturales ya mencionados, se tematizan las relaciones entre concepciones alternativas, preconcepciones y conceptos científicos.

Han sido detectadas ciertas formas de interpretar fenómenos físicos, comunes a diversas culturas. Un ejemplo: la concepción más común que se presenta sobre el movimiento es que, para que un cuerpo se mantenga en movimiento uniforme (velocidad constante), hay que aplicarle una fuerza constante de manera continua, lo cual está en abierta contradicción con la explicación que nos suministra la física newtoniana.

Una buena parte de las concepciones alternativas y las preconcepciones (ideas intuitivas) de los estudiantes, que se ponen en juego durante el aprendizaje de las ciencias, se basan en interpretaciones sustancialistas de los fenómenos naturales, que adquieren así un sentido ontológico (Thijs et al., op. cit., p. 337). Los resultados de las investigaciones sobre las ideas intuitivas ponen de manifiesto que no hay un principio organizador que dé lugar a estas ideas, éstas son construidas a partir de un conjunto de experiencias primitivas fenomenológicas que se movilizan en respuesta a situaciones específicas. Por el contrario, las interpretaciones sustancialistas permanecen en un plano más básico, más elemental y, en consecuencia, es más difícil hacerlas conscientes. Si bien las explicaciones particulares son locales y ad hoc, las convicciones ontológicas están en la base de estas explicaciones y las reactivan, porque apelan a categorías que el estudiante pone en juego para analizar el mundo y reaccionar ante él. Las convicciones ontológicas están, en su mayoría, implícitas en el razonamiento. Uno no es consciente de ellas y normalmente no las articula. Como permanecen inexploradas, pueden ser muy resistentes ante nuevas evidencias: cuando una información contradice alguna creencia superficial, ésta puede ser modificada, sin que, no obstante, cambien las convicciones ontológicas subyacentes.

Los análisis históricos y epistemológicos sobre el desarrollo de la ciencia moderna nos muestran que, en el núcleo de su fundamentación, se encuentra justamente la sustitución del pensamiento sobre la sustancia por un pensamiento sobre las relaciones. Esto último es responsable, en gran medida, de la matematización (funcional) de los modelos de la nueva ciencia. Entonces, al pretender enseñar la ciencia como hoy la conocemos, es inevitable que surjan conflictos cognitivos, pues los estudiantes tratarán de hallar el sentido de las preguntas científicas, referidas al cómo funcional, a través de sus preconcepciones y concepciones alternativas, que se refieren al por qué sustancialista. La fuerte resistencia al abandono de su conocimiento previo quizá explique la permanencia de estratos conceptuales que el sujeto 
seguirá adaptando a los contextos de sus diversas experiencias escolares.

Las preconcepciones que se refieren a la experiencia física -por ejemplo, los cuerpos más pesados caen más rápido que los más livianos- parecen universales. Las referidas en cambio a la vida, la muerte, la salud o la enfermedad están claramente marcadas por las culturas (Thijs et al., op. cit. 339). Si bien el aprendizaje de la ciencia equivale a la incorporación a una nueva cultura, lo común de las preconcepciones y concepciones alternativas ha permitido afirmar:

«No hay evidencia, hasta la fecha, que los diversos grupos culturales varíen en cuanto al repertorio de sus procesos cognitivos. Mas bien se ha visto que las diferencias culturales residen en contextos, en donde ciertos procesos particulares se tornan en sistemas funcionales. Todas aquellas preguntas sobre si distintos grupos culturales «tienen» ciertas formas de pensar y razonar han sido gradualmente sustituidas por preguntas acerca de los contextos en los que se aplican esas formas de razonamiento» (Valerie Curran, 1980, p. 328).

El lenguaje, desde luego, gravita en los procesos de aprendizaje, entre otras cosas, porque en el empleo de muchas expresiones coloquiales residen las que a veces son preconcepciones muy tenaces. Pero aun en este dominio, donde se tienen interpretaciones diametralmente opuestas como la hipótesis de Sapir-Whorf (el lenguaje moldea el pensamiento: individuos que hablan lenguas distintas piensan distinto) y la tesis de Chomsky, (sobre la existencia de una gramática universal innata), hay espacio para pensar que la manera como los humanos usamos metafóricamente el lenguaje es universal.

Este análisis que hemos esbozado sobre los estratos conceptuales y preconceptuales parece brindar un apoyo a las interpretaciones que ofrecimos anteriormente de las investigaciones realizadas por la escuela piagetiana.

La oposición entre «sentido común» y «sentido científico» nos muestra que la enseñanza de la ciencia no puede proceder, simplemente, mediante intentos de amplificación del sentido común de los estudiantes.

\section{NOTAS}

${ }^{1}$ Son procesos prácticamente simultáneos; es nuestra descripción la que genera la ilusión de linealidad en las etapas del trabajo de Piaget.

${ }^{2}$ Desde el punto de vista escolar, lo que interesa es que el estudiante pueda ampliar su campo de aplicación de los principios de conservación. En ese sentido, la instrucción tiene un impacto central sobre el desarrollo intelectual del estudiante.

${ }^{3}$ Para la escuela, de nuevo, lo importante debe ser que el estudiante pueda reconocer, cada vez de manera más sólida, las situaciones en donde tal forma de razonamiento es aplicable.

${ }^{4}$ Tomemos un ejemplo para ilustrar la idea de capacidad explicativa de una teoría. Newton postuló la existencia de una ley de gravitación universal mediante la cual pudo reconstruir el conocimiento físico de su época y, muy importante, pudo explicar hechos que hasta entonces escapaban a las explicaciones de las teorías anteriores. Después de dar estas explicaciones, ¿podemos insistir en el carácter hipotético de la ley de gravitación, para degradarla? Más bien lo que se hace es reconocer la pertinencia de dicha hipótesis, que se ha convertido en principio explicativo y organizativo de la dinámica física.

${ }^{5}$ Entendemos cultura como el complejo de características distintivas de orden espiritual, material, intelectual y emocional que caracteriza a una sociedad o grupo.

${ }^{6}$ Sugerimos al lector el estudio del capítulo IX (Ciencia, psicogénesis e ideología) de Piaget y García (1982).

\section{REFERENCIAS BIBLIOGRÁFICAS}

BISHOP, A. (1988). Mathematical Enculturation. Dordrecht: Kluwer Academic Publishers.

BRINGUIER, J.C.(1985). Conversaciones con Piaget. Barcelona: Gedisa.

DASEN, P. (1988). Between the Universal and the Specific: The Contribution of the cross-cultural approach, Archives de Psychologie, 56, pp. 265-269.
DRIVER, R., ASOKO, H., LEACH, J., MORTIMER, E. y SCOT, P. (1994). Constructing Scientific Knowledge in the Classroom, Educational Researcher, 23(7), pp. 5-12.

IFRAH, G. (1994). Histoire Universelle des Chiffres, 2 vols. París: Robert Laffont.

HAMELIN, D. y VONECHE, J. (eds.) (1996). Agir et construire. Aux origines de la connaissance chez l'enfant et le savant. 
Ginebra: Editions FPSE, Université de Genève, Musée d'ethnographie de Genève.

LEWIN, P. (1995). The social already inhabits the epistemic, en Steffe, L. Gale, J. (eds.). Constructivism in Education, pp. 423-432. Nueva Jersey: L. Erlbaum Associates.

MORENO, L. (1996). La epistemología genética: una interpretación, Educación Matemática, 8(3), pp. 5-23.

MORENO, L. y SACRISTÁN, A. (1995). On Visual and Symbolic Representations, en Sutherland, R. y Mason, J. (eds.), Exploiting Mental Imagery with Computers. Springer-Verlag.

MORENO, L. y WALDEGG, G. (1992). Constructivismo y Educación Matemática, Educación Matemática, 4(2), pp. 7-15.

PIAGET, J. (1925). Le development de la pensée de l'enfant, ProJuventute, 6, pp. 464-469.
PIAGET, J. (1929). Les deux directions de la pensée scientifique, Archives des sciences physiques et naturelles, año 134, periodo 5, vol. 11, pp. 145-162

PIAGET, J. (1988). Sabiduría e ilusiones de la filosofía. Barcelona: NeXos.

PIAGET, J. y GARCÍA, R. (1982). Psicogénesis e historia de la ciencia. México: Siglo XXI.

THIJS y VAN DER BERG (1995). Cultural Factors in the Origin and Remediation of Alternative Conceptions in Physics, Science and Education, 4, pp. 317-347.

VALERIE CURRAN, H. (1980): Cross-Cultural Perspectives on Cognition, en Claxton, G. (ed.), Cognitive Psychology, New Directions, pp. 300-334. Londres: Routledge y Kegan.

VYGOTSKY,L. (1995). Pensamiento y lenguaje. (Ed. revisada y editada por Kozulin, A.). Madrid: Paidós.

WERTSCH, J. (1985). Vygotsky and the Social Formation of Mind. Cambridge, MA: Harvard Univ. Press.

[Artículo recibido en junio de 1997 y aceptado en junio de 1998.] 